\title{
Source Code Security Analysis Tool Functional Specification Version 1.0
}

\author{
Paul E. Black \\ Michael Kass \\ Michael Koo
}

Software Diagnostics and Conformance Testing Division Information Technology Laboratory National Institute of Standards and Technology Gaithersburg, MD 20899

May 2007

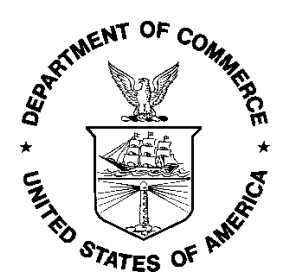

U.S. Department of Commerce Carlos M. Gutierrez, Secretary

Technology Administration Robert Cresanti, Under Secretary of Commerce for Technology National Institute of Standards and Technology William Jeffrey, Director 


\begin{abstract}
:
Software assurance tools are a fundamental resource for providing an assurance argument for today's software applications throughout the software development lifecycle. Some tools analyze software requirements, design models, source code, or executable code to help determine if an application is secure. This document specifies the behavior of one class of software assurance tool: the source code security analyzer. Because many software security weaknesses today are introduced at the implementation phase, using a source code security analyzer should help assure that software doesn't have many security vulnerabilities. This specification defines a minimum capability to help software professionals understand how a tool will meet their software security assurance needs.
\end{abstract}

\title{
Keywords:
}

Homeland security; software assurance tools; source code analysis; vulnerability.

\section{Errata to this version: \\ None}

Any commercial product mentioned is for information only. It does not imply recommendation or endorsement by NIST nor does it imply that the products mentioned are necessarily the best available for the purpose. 
Table of Contents

1 Introduction

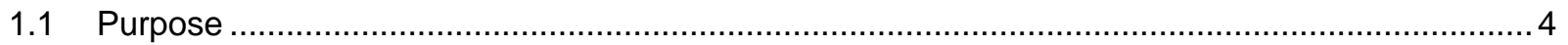

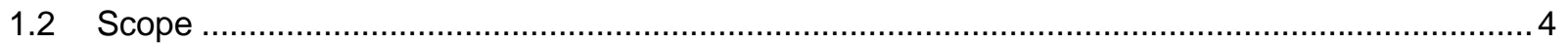

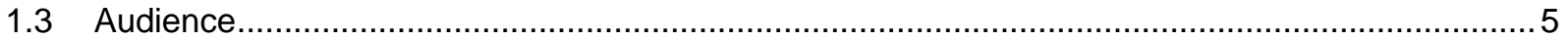

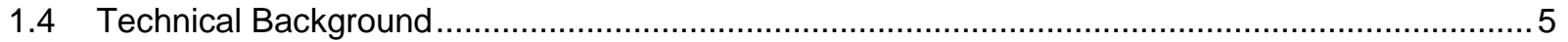

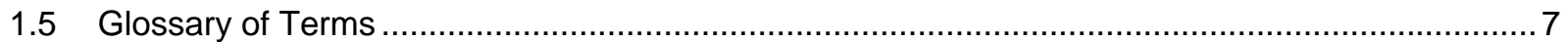

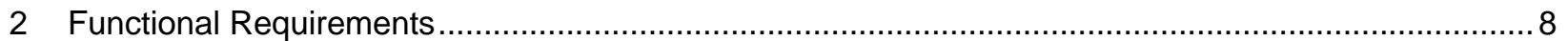

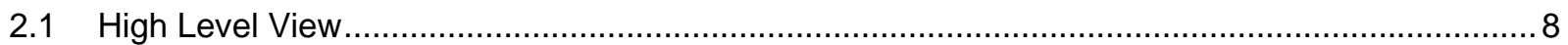

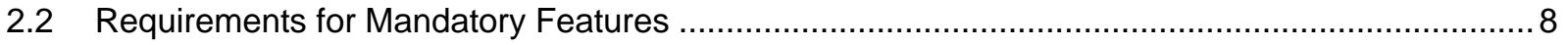

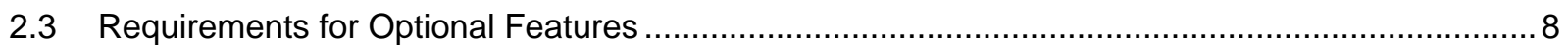

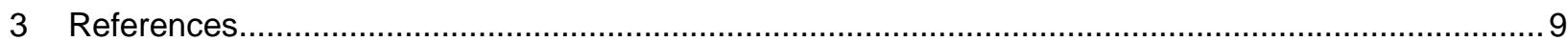

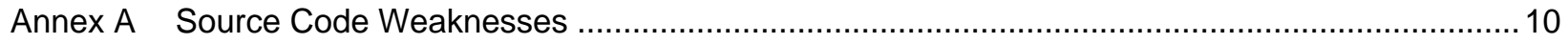

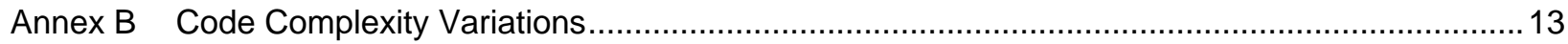




\section{Introduction}

\subsection{Purpose}

The National Institute of Standards and Technology (NIST) is working with the U.S. Department of Homeland Security National Cybersecurity Division to improve the state of the practice in software assurance. Through the development of tool functional specifications, test suites and tool metrics, the NIST Software Assurance Metrics and Tool Evaluation, or SAMATE, project aims to better quantify the state of the art for different classes of software security assurance tools.

Source code security analysis tools scan a textual (human readable) version of source files that comprise a portion or all of an application program. These files may contain inadvertent or deliberate weaknesses that could lead to security vulnerability in the executable version of the application program. This document specifies a set of functional feature requirements for a source code security analysis tool or set of tools, including a list of common code weaknesses that account for many of today's vulnerabilities.

This specification, together with the corresponding test plan and test suite, serves as a guide to measuring the capability of source code security analysis tools against this set of weaknesses. There are many useful tools that do not attempt to identify all of the weaknesses listed in this specification. The goal of this specification is not to prescribe the features and functions that all source code security analysis tools must have. The goal is to identify code weaknesses that significantly affect the security of software applications today and provide a user of such tools with a way to measure if, and how well a tool, or combination of tools, identifies these particular weaknesses.

We plan for the next version of this specification to include more weaknesses, explicitly apply to tools that only identify a subset of weaknesses, have more precise and substantiated metrics, and other enhancements.

Use of a tool or toolkit that complies with this specification does not guarantee the code will be free of weaknesses. It does however provide a tool user with knowledge that their tool solution covers some of the most prevalent and highly exploitable security weaknesses.

\subsection{Scope}

This specification is limited to software tools that examine source code files for security weaknesses and potential vulnerabilities. Tools that scan other artifacts, like requirements, bytecode or binary code, and tools that dynamically execute code are outside the scope. Annex A of this document, Source Code Weaknesses, specifically addresses $\mathrm{C}, \mathrm{C}++$, and Java source code.

We start with these three because they are the languages in which most of today's vulnerabilities have been identified and on which most source code security analysis tools focus. These weaknesses may exist in other languages as well.

As an aside, there are languages that are, by design, more suitable for secure programming. Such languages entirely preclude many common weaknesses and minimize or expose others. Choosing such languages mitigates many security risks.

This document specifies minimum functionality only. Critical production tools should have capabilities far beyond those indicated here. Many important attributes, like compatibility with integrated development environments or IDEs and ease of use, are not addressed.

The misuse or proper use of a tool is outside the scope of this specification.

The issues and challenges in engineering secure systems and their software are outside the scope of this specification. 


\subsection{Audience}

The target audience for this specification is users and evaluators of source code security analysis tools. It may also be useful to source code security analysis and software assurance researchers, and developers of source code security analysis tools.

\subsection{Technical Background}

This section gives some technical background, defines terms we use in this specification, explains how concepts designated by those terms are related, and details some challenges in source code analysis for security assurance.

\section{The Role of Source Code Analysis in Software Assurance}

No amount of analysis and patching can imbue software with high levels of security or quality or correctness or other important properties. Such properties must be designed in and built in. Good choice of language, platform, and discipline are worth orders of magnitude more than reactive efforts. Nevertheless testing or examination of code has benefits in some situations.

For instance, to determine how different methods or processes affect the quality of the resultant code, the code can be examined. If the origin of code has limited visibility, testing or static analysis are the only ways to gain higher assurance. Existing, legacy code must be examined to assess its quality and determine what, if any, remediation is needed.

Testing, or dynamic analysis, has the advantage of examining the behavior of software in operation. In contrast, only static analysis can be expected to find malicious trapdoors. Analysis of binary or executable code, including bytecode, avoids assumptions about compilation or source code semantics. Only the binary may be available for libraries or purchased software. However, source code security analysis can give developers feedback on better practices.

Remediation is often done in source code. Analysis of higher-level constructs, such as models, designs, use cases, or requirements documents, is possible, too. However, these higher-level artifacts often lack rigor and rarely reflect all the critical detail in source code implementations. Thus static analysis of source code is a reasonable place to work for higher software assurance.

\section{Terms Used in This Specification}

Often, different terms are used to refer to the same concept in software assurance and security literature. Different authors may use the same term to refer to different concepts. For the purposes of this document, the following terms and definitions apply. To begin, any event that is a violation of a particular system's explicit (or implicit) security policy is a security failure, or simply, failure. For example, if an unauthorized person gains "root" or "admin" privileges, security has failed. Again if unauthorized people can read Social Security numbers from your web site, security has failed.

A vulnerability is a property of system security requirements, design, implementation, or operation that could be accidentally triggered or intentionally exploited and result in a security failure. (After [SP800-27]) In our model the source of any failure is a latent vulnerability. In other words, if there is a failure, there must have been a vulnerability. A vulnerability is the result of one or more weaknesses in requirements, design, implementation, or operation.

In the unauthorized privileges example above, the combination of the two weaknesses of allowing weak passwords and of not locking out an account after repeated password mismatches allow the vulnerability. This vulnerability can be exploited by a brute force attack to cause the failure of an unauthorized person gaining elevated privileges. An SQL injection vulnerability might be exploited several different ways to produce different failures, such as dropping a table or revealing all its contents. If spyware can steal a user's password, it is a vulnerability. But it may be hard to attribute the vulnerability to a few lines of code that can be "fixed." Spyware typically exploits system weaknesses, which require changes at the system level.

Sometimes a weakness can never result in a failure, in which case it is not exploitable and not a vulnerability. Such a weakness might be masked by another part of the software or might only cause a 
failure in combination with another weakness. Thus we use the term "weakness" instead of "flaw" or "defect."

For several reasons no tool can correctly determine in every conceivable case whether or not a piece of code has a vulnerability. First, a weakness may result in a vulnerability in one environment, but not in another. Second, Rice proved that no algorithm can correctly decide in every case whether or not a piece of code has a property, such as a weakness. Third, practical analysis algorithms have limits because of performance and intellectual investment. Some vulnerabilities can only be identified if a tool performs inter-file, inter-procedural, or flow-sensitive analysis of the code. Each different code complexity, such as fixed or variable loops, memory indexing nested within indexing, local vs. global scope, and others listed in Annex B, may require additional analytical capabilities. Deliberate obfuscation with convoluted code structures makes the analysis even harder. Fourth, a tool may not have "rules" to find all known vulnerabilities. Worse, new exploits are being invented and new vulnerabilities recognized all the time.

Since no tool can be omniscient, a tool may be written to be cautious and report questionable constructs. Some of those reports may turn out to be false alarms or false positives. To reduce wasting users' time on false alarms, a tool may be written to only report constructs that are (almost) certainly vulnerabilities. In this case it may miss some vulnerabilities. A missed vulnerability is called a false negative. A tool may do a more detailed analysis, which is computationally intensive, to reduce both false alarms and missed vulnerabilities. The ideal is a tool that reports all real vulnerabilities (no false negatives) with no false alarms. Although this is impossible even in theory, tools may use a combination of approaches to balance performance, false alarms, and missed vulnerabilities. Since a failure only takes one vulnerability, the requirements have a tone of catching all weaknesses. Practical considerations require the false positive rate [Fleiss] to be acceptably low for the domain.

A tool may grade weaknesses according to severity, potential for exploit, certainty that they are vulnerabilities, etc. Ultimately people must analyze the tool's report and the code then decide

- which reported items are not true vulnerabilities,

- which items are acceptable risks and will not be mitigated, and

- which items to mitigate, and how to mitigate them.

To save analysis time in later runs, some tools allow the user to suppress weakness instances so they are not reported again. 


\subsection{Glossary of Terms}

This glossary provides descriptions for terms used in this document.

\begin{tabular}{|l|l|}
\hline Name & Description \\
\hline false negative & $\begin{array}{l}\text { When a tool does not report a weakness where one is present. If } \\
\text { the tool does not claim to identify a certain class of weakness, not } \\
\text { reporting a weakness of that class is not a false negative. }\end{array}$ \\
\hline false positive & When a tool reports a weakness where no weakness is present. \\
\hline false positive rate & $\begin{array}{l}\text { The number of false positives divided by the sum of the number of } \\
\text { false positives and the number of true positives. }\end{array}$ \\
\hline flow-sensitive analysis & $\begin{array}{l}\text { Analysis of a computer program that takes into account the flow of } \\
\text { control. }\end{array}$ \\
\hline inter-file analysis & $\begin{array}{l}\text { Analysis of code residing in different files that have procedural, } \\
\text { data, or other interdependencies. }\end{array}$ \\
\hline inter-procedural analysis & $\begin{array}{l}\text { Analysis between calling and called procedures within a computer } \\
\text { program. }\end{array}$ \\
\hline security failure & $\begin{array}{l}\text { Any event that is a violation of a particular system's explicit or } \\
\text { implicit security policy. }\end{array}$ \\
\hline security vulnerability & $\begin{array}{l}\text { A property of system requirements, design, implementation, or } \\
\text { operation that could be accidentally triggered or intentionally } \\
\text { exploited and result in a security failure. }\end{array}$ \\
\hline source code & $\begin{array}{l}\text { A series of statements written in a human-readable computer } \\
\text { programming language. }\end{array}$ \\
\hline tainted input & $\begin{array}{l}\text { Input data that has not been examined or sanitized prior to use by } \\
\text { an application. }\end{array}$ \\
\hline weakness suppression system & $\begin{array}{l}\text { When a tool reports a weakness where one is present. } \\
\text { reported by the tool in subsequent scans. }\end{array}$ \\
\hline
\end{tabular}




\section{Functional Requirements}

\subsection{High Level View}

Informally, what does a source code security analysis tool or tool set do? At a minimum the tool(s) should:

- Identify a select set of classes of software security weaknesses in source code.

- Report the security weaknesses that it identifies, what kind of weakness each one is, and where each one is located.

- Not have many false positives.

Optionally a tool should:

- Produce a report compatible with other tools.

- Allow weaknesses to be suppressed by the user.

- Use standard names for weakness classes.

\subsection{Requirements for Mandatory Features}

To meet a minimum capability, a source code security analysis tool or set of tools must be able to accomplish the tasks described below. The tool(s) shall:

SCSA-RM-1: Identify all of the classes of weaknesses listed in Annex A.

SCSA-RM-2: Textually report any weaknesses that it identifies.

SCSA-RM-3: For any identified weaknesses in classes listed in Annex A, report the class using a semantically equivalent name.

SCSA-RM-4: Report the location of any weaknesses by providing the directory path, file name and line number.

SCSA-RM-5: Identify weaknesses despite the presence of the coding complexities listed in Annex B.

SCSA-RM-6: Have an acceptably low false positive rate.

\subsection{Requirements for Optional Features}

The following requirements apply to optional tool features. If the tool supports an optional feature, then the requirement for that feature applies, and the tool can be tested against it. A specific tool might optionally provide none, some, or all of the features described by these requirements. Optionally, the tool(s) shall:

SCSA-RO-1: Produce an XML-formatted report.

SCSA-RO-2: Not report a weakness instance that has been suppressed.

SCSA-RO-3: Use the Common Weakness Enumeration [CWE] name of the weakness class it reports. 


\section{References}

[CVE] Common Vulnerability and Exposures, the MITRE Corporation, http://cve.mitre.org/

[CWE] Common Weakness Enumeration, the MITRE Corporation, http://cwe.mitre.org/

[Fleiss] Fleiss, J. L. (1981). Statistical Methods for Rates and Proportions, $2^{\text {nd }}$ ed., John Wiley and Sons, New York, pp 4-8.

[Kratkiewicz] Kratkiewicz, K. (2005). Evaluating Static Analysis Tools for Detecting Buffer Overflows in C Code, Master's Thesis, Harvard University, Cambridge, MA, 285 pages. http://www.II.mit.edu/IST/pubs/KratkiewiczThesis.pdf

[SP800-27] Engineering Principles for Information Technology Security (A Baseline for Achieving Security), NIST SP 800-27, Revision A, June 2004. http://csrc.nist.gov/publications/nistpubs/

[XML] Extensible Markup Language, World Wide Web Consortium (W3C), http://www.w3.org/XML/ 


\section{Annex A Source Code Weaknesses}

The classes of source code weaknesses listed in this table represent a "base set" of code weaknesses. Criteria for selection of weaknesses include:

- Found in existing code today - The weaknesses are found in existing software applications.

- Recognized by tools today - Tools today are able to identify these weaknesses in source code and identify their associated file names and line numbers.

- Likelihood of exploit is medium to high - The weakness is fairly easy for a malicious user to recognize and to exploit.

For each weakness, this table contains the CWE name and identifier number, a short description, the relevant language(s), and the code complexities that apply. For other relevant information, such as likelihood of exploitation, instances in the common vulnerability and exposures list [CVE], background, consequences, and remediation, please see [CWE].

\begin{tabular}{|c|c|c|c|c|}
\hline Name & CWE ID & Description & Language(s) & Relevant Complexities \\
\hline \multicolumn{5}{|l|}{ Input Validation } \\
\hline Basic XSS & 80 & $\begin{array}{l}\text { Unfiltered input is passed } \\
\text { to a web application that in } \\
\text { turn passes that data back } \\
\text { to another client in the } \\
\text { form of a malicious script. }\end{array}$ & C,C++, Java & $\begin{array}{l}\text { taint, scope, address alias level, } \\
\text { container, local control flow, loop } \\
\text { structure, buffer address type }\end{array}$ \\
\hline $\begin{array}{l}\text { Resource } \\
\text { Injection }\end{array}$ & 99 & $\begin{array}{l}\text { Unfiltered input is used in } \\
\text { an argument to a resource } \\
\text { operation function. }\end{array}$ & C, $\mathrm{C}++$, Java & $\begin{array}{l}\text { taint, scope, address alias level, } \\
\text { container, local control flow, loop } \\
\text { structure, buffer address type }\end{array}$ \\
\hline $\begin{array}{l}\text { OS Command } \\
\text { Injection }\end{array}$ & 78 & $\begin{array}{l}\text { Unfiltered input is used in } \\
\text { an argument to a system } \\
\text { operation execution } \\
\text { function. }\end{array}$ & C, C++, Java & $\begin{array}{l}\text { taint, scope, address alias level, } \\
\text { container, local control flow, loop } \\
\text { structure, buffer address type }\end{array}$ \\
\hline SQL Injection & 89 & $\begin{array}{l}\text { Unfiltered input is used in } \\
\text { an argument to a SQL } \\
\text { command calling function. }\end{array}$ & C, C++, Java & $\begin{array}{l}\text { taint, scope, address alias level, } \\
\text { container, local control flow, loop } \\
\text { structure, buffer address type }\end{array}$ \\
\hline \multicolumn{5}{|l|}{ Range Errors } \\
\hline Stack overflow & 121 & $\begin{array}{l}\text { Input is used in an } \\
\text { argument to the creation } \\
\text { or copying of blocks of } \\
\text { data beyond the fixed } \\
\text { memory boundary of a } \\
\text { buffer on the stack. }\end{array}$ & C, C++ & All \\
\hline Heap overflow & 122 & $\begin{array}{l}\text { Input is used in an } \\
\text { argument to the creation } \\
\text { or copying of blocks of } \\
\text { data beyond the fixed } \\
\text { memory boundary of a } \\
\text { buffer in the heap portion } \\
\text { of memory. }\end{array}$ & C, C++ & All \\
\hline
\end{tabular}




\begin{tabular}{|c|c|c|c|c|}
\hline $\begin{array}{l}\text { Format string } \\
\text { vulnerability }\end{array}$ & 134 & $\begin{array}{l}\text { Unfiltered input is used in } \\
\text { a string used to format } \\
\text { data in the printf() style of } \\
\text { C/C++ functions. }\end{array}$ & C, C++ & $\begin{array}{l}\text { taint, scope, address alias level, } \\
\text { container, local control flow, loop } \\
\text { structure, buffer address type }\end{array}$ \\
\hline $\begin{array}{l}\text { Improper Null } \\
\text { Termination }\end{array}$ & 170 & $\begin{array}{l}\text { The software does not } \\
\text { properly terminate a string } \\
\text { or array with a null } \\
\text { character or equivalent } \\
\text { terminator }\end{array}$ & C, C++ & $\begin{array}{l}\text { taint, scope, address alias level, } \\
\text { container, local control flow, loop } \\
\text { structure, buffer address type }\end{array}$ \\
\hline \multicolumn{5}{|l|}{ API Abuse } \\
\hline Heap Inspection & 244 & $\begin{array}{l}\text { Using realloc() to resize } \\
\text { buffers that store sensitive } \\
\text { information can leave the } \\
\text { sensitive information } \\
\text { exposed to attack because } \\
\text { it is not removed from } \\
\text { memory. }\end{array}$ & C, $\mathrm{C}++$ & $\begin{array}{l}\text { taint, scope, address alias level, } \\
\text { container, local control flow, loop } \\
\text { structure, buffer address type }\end{array}$ \\
\hline $\begin{array}{l}\text { Often Misused: } \\
\text { String } \\
\text { Management }\end{array}$ & 251 & $\begin{array}{l}\text { Some string manipulation } \\
\text { functions can be exploited } \\
\text { through their input to } \\
\text { produce buffer overflows. }\end{array}$ & C, C++ & $\begin{array}{l}\text { taint, scope, address alias level, } \\
\text { container, local control flow, loop } \\
\text { structure, buffer address type }\end{array}$ \\
\hline \multicolumn{5}{|l|}{ Security Features } \\
\hline $\begin{array}{l}\text { Hard-Coded } \\
\text { Password }\end{array}$ & 259 & $\begin{array}{l}\text { Hard-coded data is passed } \\
\text { as an argument to a login } \\
\text { function. }\end{array}$ & C/C++, Java & $\begin{array}{l}\text { scope, address alias level, } \\
\text { container, local control flow, loop } \\
\text { structure, buffer address type }\end{array}$ \\
\hline \multicolumn{5}{|l|}{ Time and State } \\
\hline $\begin{array}{l}\text { Time-of-check } \\
\text { Time-of-use race } \\
\text { condition }\end{array}$ & 367 & $\begin{array}{l}\text { Between the time in which } \\
\text { a given resource (or its } \\
\text { reference) is checked, and } \\
\text { the time that resource is } \\
\text { used, a change occurs in } \\
\text { the resource to invalidate } \\
\text { the results of the check. }\end{array}$ & C, C++, Java & asynchronous \\
\hline $\begin{array}{l}\text { Unchecked Error } \\
\text { Condition }\end{array}$ & 391 & $\begin{array}{l}\text { No action is taken after an } \\
\text { error or exception } \\
\text { condition occurs. }\end{array}$ & C, C++, Java & none \\
\hline \multicolumn{5}{|l|}{ Code Quality } \\
\hline Memory leak & 401 & $\begin{array}{l}\text { Memory is allocated, but is } \\
\text { not released after it has } \\
\text { been used. }\end{array}$ & C, C++ & $\begin{array}{l}\text { scope, address alias level, } \\
\text { container, local control flow, loop } \\
\text { structure }\end{array}$ \\
\hline $\begin{array}{l}\text { Unrestricted } \\
\text { Critical Resource } \\
\text { Lock }\end{array}$ & 412 & $\begin{array}{l}\text { A resource is "deadlocked" } \\
\text { by obtaining an exclusive } \\
\text { lock or mutex, or modifying } \\
\text { the permissions of a } \\
\text { shared resource. }\end{array}$ & C, C++, Java & asynchronous \\
\hline
\end{tabular}




\begin{tabular}{|c|c|c|c|c|}
\hline Double Free & 415 & $\begin{array}{l}\text { An attempt is made to free } \\
\text { memory using an address } \\
\text { that has previously been } \\
\text { used in a free () function } \\
\text { call. }\end{array}$ & C, C++ & $\begin{array}{l}\text { scope, address alias level, } \\
\text { container, local control flow, loop } \\
\text { structure, buffer address type }\end{array}$ \\
\hline Use After Free & 416 & $\begin{array}{l}\text { An attempt is made to } \\
\text { access the same memory } \\
\text { address previously } \\
\text { released by a call to the } \\
\text { free() function. }\end{array}$ & C, C++ & $\begin{array}{l}\text { scope, address alias level, } \\
\text { container, local control flow, loop } \\
\text { structure, buffer address type }\end{array}$ \\
\hline $\begin{array}{l}\text { Uninitialized } \\
\text { variable }\end{array}$ & 457 & $\begin{array}{l}\text { A variable is created } \\
\text { without assigning it a } \\
\text { value. It is subsequently } \\
\text { referenced in the program, } \\
\text { causing potential } \\
\text { undefined behavior or } \\
\text { denial or service. }\end{array}$ & C, C++ & $\begin{array}{l}\text { scope, address alias level, } \\
\text { container, local control flow, loop } \\
\text { structure }\end{array}$ \\
\hline $\begin{array}{l}\text { Unintentional } \\
\text { pointer scaling }\end{array}$ & 468 & $\begin{array}{l}\text { Improper mixing of pointer } \\
\text { types in an expression } \\
\text { may result in references to } \\
\text { memory beyond that } \\
\text { intended by the program. }\end{array}$ & C, $\mathrm{C}++$ & data type \\
\hline Null Dereference & 476 & $\begin{array}{l}\text { A pointer with a value of } \\
\text { NULL is used as though it } \\
\text { pointed to a valid memory } \\
\text { area. }\end{array}$ & C, $C++$ & $\begin{array}{l}\text { taint, scope, address alias level, } \\
\text { container, local control flow, loop } \\
\text { structure }\end{array}$ \\
\hline \multicolumn{5}{|l|}{ Encapsulation } \\
\hline $\begin{array}{l}\text { Leftover Debug } \\
\text { Code }\end{array}$ & 489 & $\begin{array}{l}\text { Debug code can create } \\
\text { unintended entry points in } \\
\text { an application. }\end{array}$ & C, C++, Java & none \\
\hline
\end{tabular}




\section{Annex B Code Complexity Variations}

To locate and identify source code weaknesses listed in Annex A, a source code security analysis tool must be able to find those weaknesses within the complex coding structures relevant to that weakness. A general list of these types of structures, adapted from [Kratkiewicz], is provided below. Some of the complexities are language specific (e.g. the use of pointers in C, C++), however, most exist in $\mathrm{C}$, C++ and Java. Equivalent constructs in other languages will be added, as tools for those languages are addressed in this specification.

\begin{tabular}{|c|c|c|}
\hline Complexity & Description & Enumeration \\
\hline address alias level & $\begin{array}{l}\text { level of "indirection" of buffer alias } \\
\text { using variable(s) containing the } \\
\text { address }\end{array}$ & 1 or 2 \\
\hline array address complexity & $\begin{array}{l}\text { level of complexity of the address } \\
\text { value of an array buffer }\end{array}$ & $\begin{array}{l}\text { constant, variable, linear expression, } \\
\text { nonlinear expression, function return } \\
\text { value, array content value }\end{array}$ \\
\hline array index complexity & $\begin{array}{l}\text { level of complexity of the index } \\
\text { value of an array buffer using } \\
\text { variable assignment }\end{array}$ & $\begin{array}{l}\text { constant, variable, linear expression, } \\
\text { nonlinear expression, function return } \\
\text { value, array content value }\end{array}$ \\
\hline array length/limit complexity & $\begin{array}{l}\text { level of complexity of the index of } \\
\text { an array buffer's length or limit } \\
\text { value }\end{array}$ & $\begin{array}{l}\text { constant, variable, linear expression, } \\
\text { nonlinear expression, function return } \\
\text { value, array content value }\end{array}$ \\
\hline asynchronous & asynchronous coding construct & threads, forked process, signal handler \\
\hline buffer address type & method used to address buffer & pointer, array index \\
\hline container & containing data structure & $\begin{array}{l}\text { array, struct, union, array of structs, } \\
\text { array of unions }\end{array}$ \\
\hline data type & type of data read or written & $\begin{array}{l}\text { character,integer,floating point,wide } \\
\text { character,pointer,unsigned } \\
\text { character,unsigned integer }\end{array}$ \\
\hline index alias level & $\begin{array}{l}\text { level of buffer index alias } \\
\text { indirection }\end{array}$ & 1 or 2 \\
\hline local control flow & $\begin{array}{l}\text { type of control flow around } \\
\text { weakness }\end{array}$ & $\begin{array}{l}\text { if,switch,cond,goto/label,setjmp,longjmp, } \\
\text { function pointer, recursion }\end{array}$ \\
\hline loop complexity & component of loop that is complex & initialization, test, increment \\
\hline loop iteration & type of loop iteration/termination & fixed, indefinite \\
\hline loop structure & $\begin{array}{l}\text { type of loop construct in which } \\
\text { weakness is embedded }\end{array}$ & $\begin{array}{l}\text { standard for, standard do while, standard } \\
\text { while, non standard for, non standard do } \\
\text { while, non standard while }\end{array}$ \\
\hline memory access & $\begin{array}{l}\text { type of memory access related to } \\
\text { weakness }\end{array}$ & read, write \\
\hline memory location & $\begin{array}{l}\text { type of memory location related to } \\
\text { weakness }\end{array}$ & $\begin{array}{l}\text { heap, stack, data region, BSS, shared } \\
\text { memory }\end{array}$ \\
\hline
\end{tabular}




\begin{tabular}{|l|l|l|}
\hline scope & $\begin{array}{l}\text { scope of control flow related to } \\
\text { weakness }\end{array}$ & $\begin{array}{l}\text { local, within-file/inter-procedural, within- } \\
\text { file/global, inter-file/inter-procedural, } \\
\text { inter-file/global, inter-class }\end{array}$ \\
\hline taint & type of tainting to input data & $\begin{array}{l}\text { argc/argv, environment variables, file or } \\
\text { stdin, socket, process environment }\end{array}$ \\
\hline
\end{tabular}

